\title{
Comprehensive analysis of aberrantly expressed long non-coding RNAs, microRNAs, and mRNAs associated with the competitive endogenous RNA network in cervical cancer
}

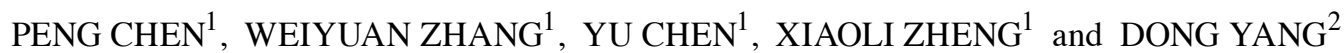 \\ ${ }^{1}$ Department of Obstetrics and Gynecology, Beijing Obstetrics and Gynecology Hospital, Capital Medical University; \\ ${ }^{2}$ Department of Obstetrics and Gynecology, Beijing Shijitan Hospital, Capital Medical University, Beijing 100000, P.R. China
}

Received June 02, 2019; Accepted March 20, 2020

DOI: $10.3892 / \mathrm{mmr} .2020 .11120$

\begin{abstract}
Cervical cancer is a common malignant disease that poses a serious health threat to women worldwide. Growing research efforts have focused on protein-coding and non-coding RNAs involved in the tumorigenesis and prognosis of various types of cancer. The potential molecular mechanisms and the interaction among long non-coding RNAs (lncRNAs), microRNAs (miRNAs), and mRNAs require further investigation in cervical cancer. In the present study, IncRNA, miRNA, and mRNA expression profiles of 304 primary tumor tissues from patients with cervical cancer and 3 solid normal tissues from The Cancer Genome Atlas (TCGA) dataset were studied via RNA sequencing (RNA-seq). Gene Ontology (GO) and Kyoto Encyclopedia of Genes and Genomes (KEGG) pathway analyses were performed using $\mathrm{R}$ package clusterProfiler to annotate the principal functions of differentially expressed (DE) mRNAs. Kaplan-Meier analysis was also conducted to investigate the effects of DElncRNAs, DEmiRNAs, and DEmRNAs on overall survival. A total of 2,255 mRNAs, 133 miRNAs, and 150 lncRNAs that were differentially expressed were identified with a threshold of $\mathrm{P}<0.05$ and Ifold change (FC) $\mid>2$. Functional enrichment analysis indicated that DEmRNAs were enriched in cancer-associated KEGG pathways. Furthermore, 255 mRNAs, 15 miRNAs, and 12 lncRNAs that were significantly associated with overall survival in cervical carcinoma were also identified. Importantly, an miRNA-mediated competitive endogenous RNA (ceRNA) network was successfully constructed based on the expression profiles of DElncRNAs and DEmRNAs. More importantly, it was found that the
\end{abstract}

Correspondence to: Professor Weiyuan Zhang, Department of Obstetrics and Gynecology, Beijing Obstetrics and Gynecology Hospital, Capital Medical University, 251 Yaojiayuan Road, Chaoyang, Beijing 100000, P.R. China

E-mail: zhangwy9921@hotmail.com

Key words: long non-coding RNA, microRNA, competing endogenous RNA, cervical cancer, expression profile
lncRNA EPB41L4A-AS1 may function as a pivotal regulator in carcinoma of the uterine cervix. Taken together, the present study has provided novel insights into investigating the potential mechanisms underlying tumorigenesis, development, and prognosis of cervical cancer, and presented new potential avenues for cancer therapeutics.

\section{Introduction}

Carcinoma of the uterine cervix is a severe malignant neoplasm that affects women globally (1). The mortality rate resulting from cervical cancer remains high, and is second only to the mortality rate of breast cancer (2). Cervical cancer is a complex disease, and in addition to infection with the well-studied human papillomavirus (HPV), many other factors contribute to the tumorigenesis and progression of neoplasms. Aberrant genes, multiparity, and smoking may exert a significant role in the risk of cervical cancer (3). Therefore, novel therapeutic and prognostic targets for cervical cancer are urgently needed.

There is increasing evidence that long non-coding RNAs (lncRNAs) and mRNAs fulfill key roles in tumorigenesis and tumor prognosis. Both IncRNAs and mRNAs may act as microRNA (miRNA) sponges that bind to miRNA response elements (MREs) to regulate gene expression (4). However, the function of IncRNAs has yet to be adequately elucidated. Increasing evidence has suggested that IncRNAs participate in various biological processes, including cell differentiation, invasion, migration, and the cell cycle (5). The carcinogenic mechanisms of lncRNAs at the molecular level, as well as their prognostic potential, are still largely unknown in cervical cancer.

MiRNAs belong to the category of short non-coding RNAs, which may be either upregulated or downregulated in malignant tumors and may regulate the expression of protein-coding RNAs via direct binding to these sequences. Similarly to lncRNAs and mRNAs, miRNAs notably influence tumor growth, invasion, and migration by acting as either oncogenes or tumor suppressor genes, and therefore may influence disease outcome in patients with cancer (6).

Thus, a large-scale analysis of lncRNA-miRNA-mRNA interactions as a competitive endogenous RNA (ceRNA) network is essential for the comprehensive understanding of the oncogenesis and prognosis of cervical cancer. To date, 
however, few studies have been published that aimed to elucidate the association among mRNAs, lncRNAs, and miRNAs through a ceRNA network in cervical cancer.

The Cancer Genome Atlas (TCGA) is a public database that provides access to genomic data from various types of cancer (7). In the present study, RNA-sequencing (RNA-seq) profiles were retrieved from TCGA to analyze genes that are dysregulated in cervical cancer. The relationship between aberrant RNA expression profiles and overall survival was also analyzed. Moreover, Gene Ontology (GO) and Kyoto Encyclopedia of Genes and Genomes (KEGG) analyses were conducted to annotate the DEmRNAs.

A total of 255 mRNAs, 15 miRNAs, and 12 lncRNAs were identified as prognostic biomarkers for patients with cervical cancer. Importantly, a miRNA-mediated lncRNA-miRNA-mRNA ceRNA network was constructed and, to the best of the authors' knowledge for the first time, the IncRNA EPB41L4A-AS1 was confirmed as a pivotal regulator in cervical carcinoma through bioinformatics analysis. Taken together, our results suggest this novel lncRNA is a potential diagnostic biomarker, contributing to our knowledge of the interactions between mRNAs and non-coding RNAs in cervical cancer.

\section{Materials and methods}

Materials and data. A total of 307 cases were retrieved from TCGA database, among which 304 were cervical squamous cell carcinoma and endocervical adenocarcinoma (CESC) primary tumor tissues, and three were solid normal tissues. RNA expression data, as well as clinical information, were obtained from TCGA website (Date of access for database: 27th August 2018). Note that medical ethics committee approval was not required for the present study.

Differential gene expression analysis. Linear Models for Microarray Data (Limma) (8) was used to identify DE genes (DEGs), comparing between primary tumor tissues and solid normal tissues. The ggplot2 (http:/ggplot.yhathq.com/) package in $\mathrm{R}$ software was employed to generate a volcano plot and to perform hierarchical clustering analysis to distinguish statistically significant DEGs. The selection criteria were $\mathrm{P}<0.05$ and Ifold changel $>2$ for DEGs.

Functional enrichment analysis. R package clusterProfiler (9) was used to conduct functional enrichment analysis, and significant enrichment in GO and KEGG pathways $(\mathrm{P}<0.01$ and $\mathrm{q}<0.01$ ) were identified. P-values were adjusted with the Benjamini-Hochberg method. A selection of DEGs that were significantly enriched in cell cycle pathways were displayed in a signal pathway diagram.

Univariate survival analysis. Kaplan-Meier (KM) analysis based on the survival package (https:/www.rdocumentation. org/packages/survival) was used for univariate survival analysis. The KM analysis divides patients into upregulated and downregulated groups according to median expression levels.

Analysis of ceRNAs network. Three criteria were used to determine the competing endogenous interactions between
lncRNA-mRNA pairs: i) the lncRNA and mRNA must share a significant number of miRNAs. A hypergeometric test was used to determine whether a lncRNA and mRNA shared a significant number of miRNAs. A newly developed algorithm spongeScan (10) was employed to predict MREs of lncRNAs. StarBase v.2.0 (11) was used to retrieve predicted and experimentally confirmed miRNA-mRNA and/or miRNA-lncRNA interactions. ii) Expression of lncRNA and mRNA must be positively correlated. There have been a number of reports of miRNAs acting as negative regulators of gene expression. If a greater number of miRNAs are occupied by lncRNAs, fewer of these miRNAs will bind to the target mRNA, thus increasing the expression level of mRNA. Therefore, expression of lncRNAs and mRNAs in a ceRNA pair should be positively correlated. iii) MiRNAs that share similar sequences should serve similar roles in regulating the expression of lncRNAs and mRNAs.

Two methods were used to determine the regulatory role of miRNAs in the lncRNAs and mRNAs:

Regulation similarity. A similarity score was defined to analyze the similarity between miRNA-lncRNA expression correlation and miRNA-mRNA expression correlation. The following formula was used:

$$
\text { Regulation similarity score }=1-\frac{1}{M} \sum_{k=1}^{M}\left\{\left.\frac{\left|\operatorname{corr}\left(m_{k}, l\right)-\operatorname{corr}\left(m_{k}, g\right)\right|}{\left|\operatorname{corr}\left(m_{k}, l\right)\right|+\left|\operatorname{corr}\left(m_{k}, g\right)\right|}\right|^{M}\right.
$$

Where $M$ indicates the total number of shared microRNAs, $\mathrm{k}$ stands for the $\mathrm{k}^{\text {th }}$ shared miRNA, and $\operatorname{corr}(m k, l)$ and $\operatorname{corr}(m k, g)$ represent the Pearson correlation between the kth miRNA and lncRNA and the kth miRNA and mRNA, respectively.

Sensitivity correlation. Sensitivity correlation, previously defined by Paci et al (12), was used to examine whether the interaction between mRNA and IncRNA is mediated by miRNA in the IncRNA-miRNA-mRNA triplet. The average of all triplets of a lncRNA-mRNA pair and their shared miRNAs was considered as the sensitivity relevance between a selected mRNA and lncRNA:

Sensitivity correlation $=\operatorname{corr}(l, g)-\frac{1}{M} \sum_{k=1}^{M} \frac{\operatorname{corr}(l, g)-\operatorname{corr}\left(m_{k}, l\right) \operatorname{corr}\left(m_{k}, g\right)}{\sqrt{1-\operatorname{corr}\left(m_{k}, l\right)^{2}} \sqrt{1-\operatorname{corr}\left(m_{k}, g\right)^{2}}}$

Where $M$ indicates the total number of shared miRNAs, $k$ stands for the $\mathrm{k}^{\text {th }}$ shared miRNA, $\operatorname{corr}(l, g), \operatorname{corr}(m k, l)$, and $\operatorname{corr}(m k, g)$ represent the Pearson correlation among the lncRNA and the protein coding gene, the $\mathrm{k}^{\text {th }}$ miRNA and lncRNA, and the $\mathrm{k}^{\text {th }}$ miRNA and mRNA, respectively. The network was further filtered by hyperP-value $<0.05$ and corP-value $<0.05$. The ceRNA network was visualized with Cytoscape (v.3.6.1) (13). The Cytoscape plug-in called 'cytoHubba' (14) was used to explore hub nodes. The maximal clique centrality (MCC) algorithm of cytoHubba was used to identify hub nodes.

\section{Results}

Identification of DEmRNAs and DEmiRNAs. RNA expression profiles of 304 CESC and three solid normal tissues were obtained from TCGA database. DE analysis of RNA expres- 
Table I. Top 10 DElncRNAs in CESC tissues compared with normal samples.

\begin{tabular}{lcccc}
\hline lncRNA & Expression change & Log2 FC & P-value \\
\hline EMX2OS & Downregulation & -6.54 & $2.93 \times 10^{-20}$ & $3.19 \times 10^{-18}$ \\
MAGI2-AS3 & Downregulation & -4.70 & $1.27 \times 10^{-29}$ & $4.28 \times 10^{-27}$ \\
MIR100HG & Downregulation & -4.36 & $6.34 \times 10^{-16}$ & $4.17 \times 10^{-14}$ \\
DIO3OS & Downregulation & -4.17 & $2.50 \times 10^{-08}$ & $4.99 \times 10^{-07}$ \\
DNM3OS & Downregulation & -3.91 & $5.65 \times 10^{-15}$ & $2.16 \times 10^{-13}$ \\
LINC01451 & Upregulation & 6.04 & $6.38 \times 10^{-4}$ & $3.81 \times 10^{-3}$ \\
FAM83A-AS1 & Upregulation & 6.28 & $3.89 \times 10^{-4}$ & $4.32 \times 10^{-3}$ \\
C50rf66-AS1 & Upregulation & 6.62 & $9.30 \times 10^{-4}$ & $2.83 \times 10^{-3}$ \\
AC245041.1 & Upregulation & 6.82 & $3.93 \times 10^{-4}$ \\
AL049555.1 & Upregulation & 7.47 & $5.88 \times 10^{-3}$ \\
\hline
\end{tabular}

CESC, cervical squamous cell carcinoma and endocervical adenocarcinoma; DE, differentially expressed; FC, fold change; FDR, false discovery rate.

A

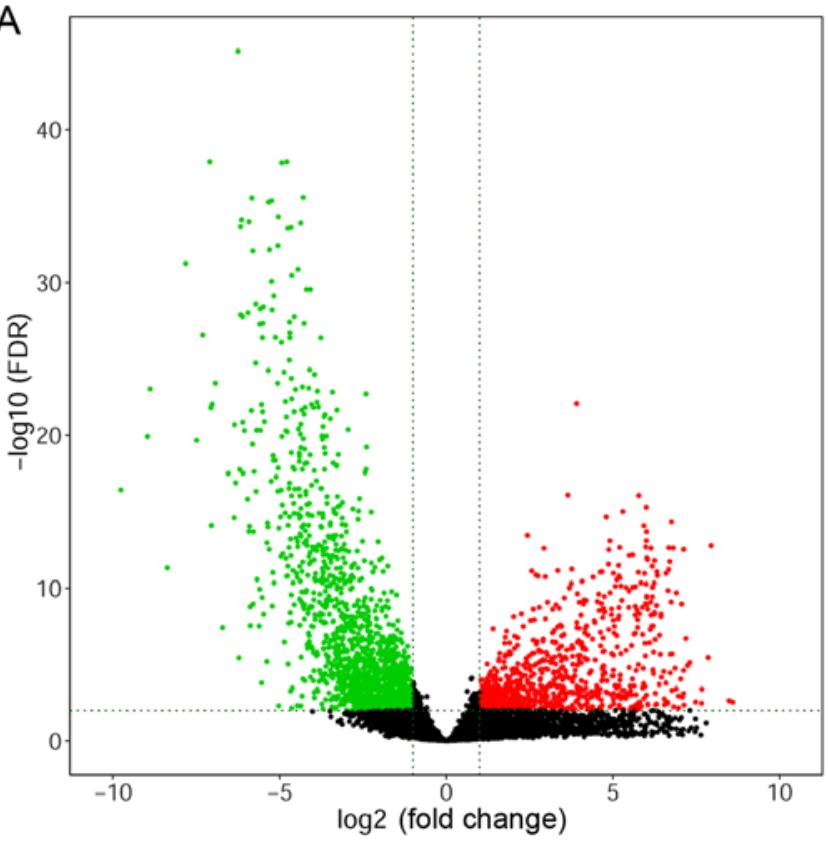

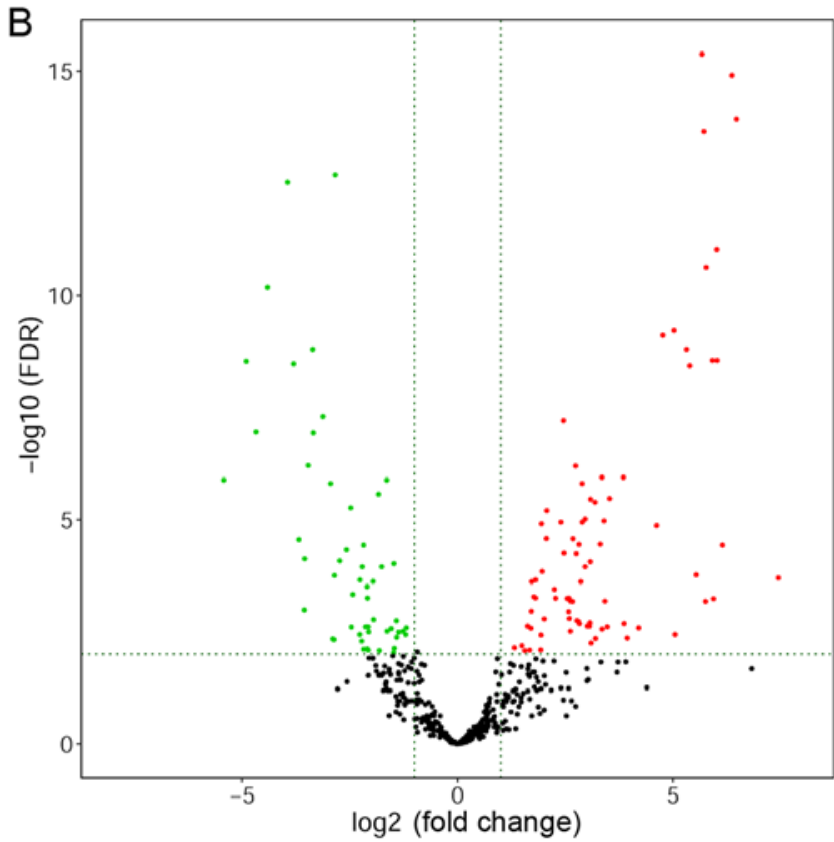

Figure 1. Volcano maps of DEGs. Red and green dots represent DEGs with high and low expression, respectively. (A) Volcano map of DEmRNAs. (B) Volcano map of DEmiRNAs. DEG, differentially expressed genes; FDR, false discovery rate.

sion levels in CESC tissues compared with those of normal samples was performed. In total, 2,255 DEmRNAs and 133 DEmiRNAs were confirmed with cut-off values of $\mathrm{P}<0.05$ and $\mid \mathrm{FCl}>2$. There were $796 \mathrm{DEmRNAs}$ that were significantly upregulated, and 1,459 DEmRNAs that were strongly downregulated. Moreover, 80 DEmiRNAs were upregulated, and 53 were downregulated based on the differential expression profiles. FOXA1, PITX1, OTX1, SFN, and NMU were the top five upregulated mRNAs, whereas DES, ACTG2, CNN1, MYH11, and PTGIS were the top five downregulated mRNAs. Additionally, the top five most highly expressed miRNAs were miR-205-5p, miR-141-3p, miR-141-5p, miR-203b-3p, and miR-200c-5p, whereas miR-204-5p, miR-133a-3p, miR-1-3p, miR-145-5p, and miR-10b-5p were the top five most downregulated miRNAs. Volcano plots were subsequently generated to identify the DEGs with statistically significant differences, comparing between primary tumors and normal tissues (Fig. 1). Heat maps of hierarchical clustering analysis were also generated for the DEGs (Fig. 2).

Differentially expressed lncRNAs (DElncRNAs) and survival analysis in CESC. A total of 150 DElncRNAs were identified to have dysregulated expression profiles in CESC tissues compared with those in normal samples. Among them, 60 DElncRNAs were upregulated and 90 DElncRNAs were significantly downregulated. The top 10 DElncRNAs are presented in Table I. Subsequently, the association between DElncRNAs and the prognosis of patients with CESC using KM analysis was studied. This analysis revealed that 12 DElncRNAs were clearly related to overall 

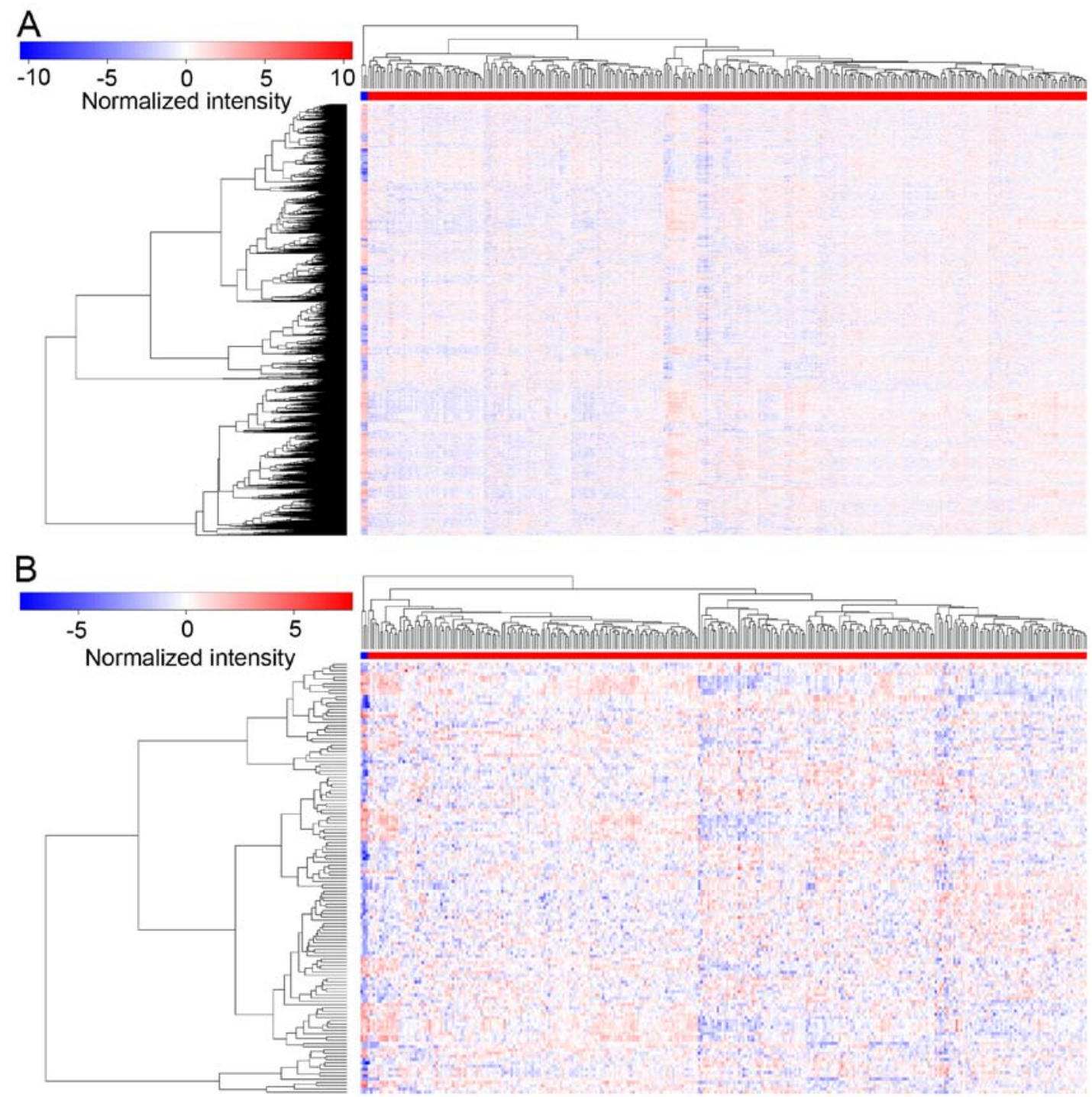

Figure 2. Heatmap of differentially expressed profiles in CESC. In the heatmap, the blue strip represents normal tissues and the red strip represents tumor tissues. Gradient colors from blue to red indicate genes from low- to high expression. (A) Heatmap of DEmRNAs. A total of 2,255 DEmRNAs were confirmed: 796 DEmRNAs were significantly upregulated, and 1,459 DEmRNAs were downregulated. (B) Heatmap of DEmiRNAs. A total of 133 DEmiRNAs were confirmed: 80 DEmiRNAs were significantly upregulated, and 53 DEmiRNAs were markedly downregulated. CESC, cervical squamous cell carcinoma and endocervical adenocarcinoma; DE, differentially expressed.

survival. The overall survival was positively associated with 9 DElncRNAs (AC008771.1, AC015871.3, AC016773.1, AC093278.2, AL662844.4, EMX2OS, ILF3-AS1, TFAP2A-AS1, and ZSCAN16-AS1) (Fig. 3), and negatively associated with 3 DElncRNAs (AC019069.1, BAIAP2-AS1, and CASC15).

GO and KEGG pathway annotation of DEmRNAs. To explore the potential functions of aberrantly expressed mRNAs that may be associated with the tumorigenesis and development of CESC, GO and KEGG pathway analyses were conducted using the $\mathrm{R}$ package clusterProfiler. The annotations of enriched GO terms were related to cell division, cell proliferation, cellular protein metabolic process, and cell differentiation. The top 30 significantly enriched GO terms are shown in Fig. 4A. KEGG enrichment indicated that the DEmRNAs were remarkably well-enriched in tumor-associated pathways; including focal adhesion, cell cycle, cellular senescence, DNA replication, p53 signaling pathway, Fanconi anemia pathway, homologous recombination, and base excision repair. The top 10 enriched pathways are shown in Fig. 4B. It was noted that DEmRNAs enriched in the biological processes of the top $10 \mathrm{GO}$ terms were mainly associated with the cell cycle in KEGG pathways. Therefore, the DEmRNAs of CESC are depicted in the signal pathway diagram of the cell cycle. A plot describing the cell cycle and involvement of DEmRNAs is shown in Fig. 5. Gradient colors are used to indicate high- or low-expression genes. The results of the functional enrichment analyses suggest that the DEmRNAs may participate in oncogenicity and tumor progression in CESC through regulating relevant biological processes and critical pathways.

Assessment of DEmRNAs and DEmiRNAs associated with overall survival. KM curves were generated to analyze the relationship between RNA expression and overall survival in patients with CESC. In the present study, 255 DEmRNAs 

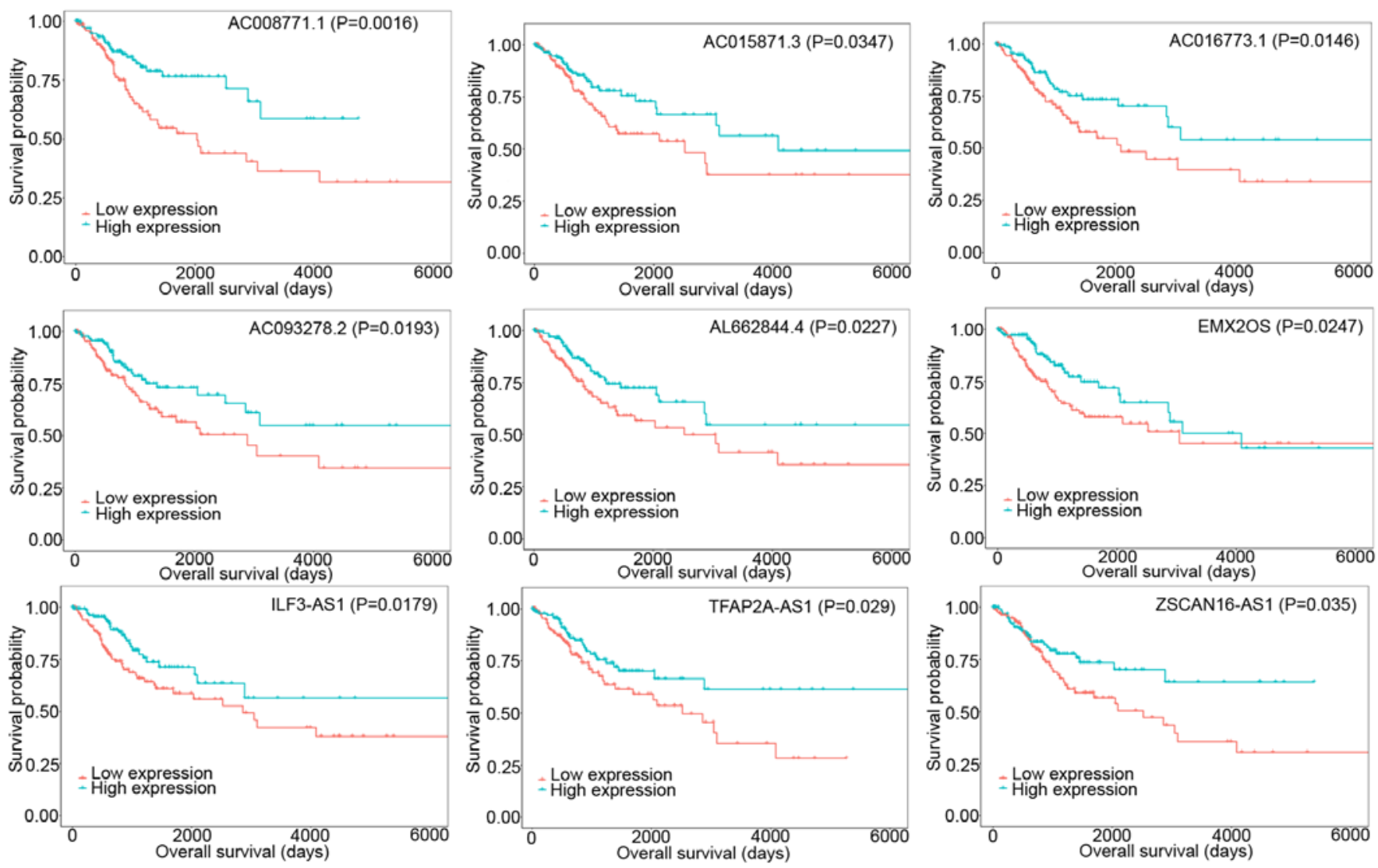

Figure 3. Kaplan-Meier survival curves of 9 DElncRNAs were positively associated with overall survival. DE, differentially expressed.
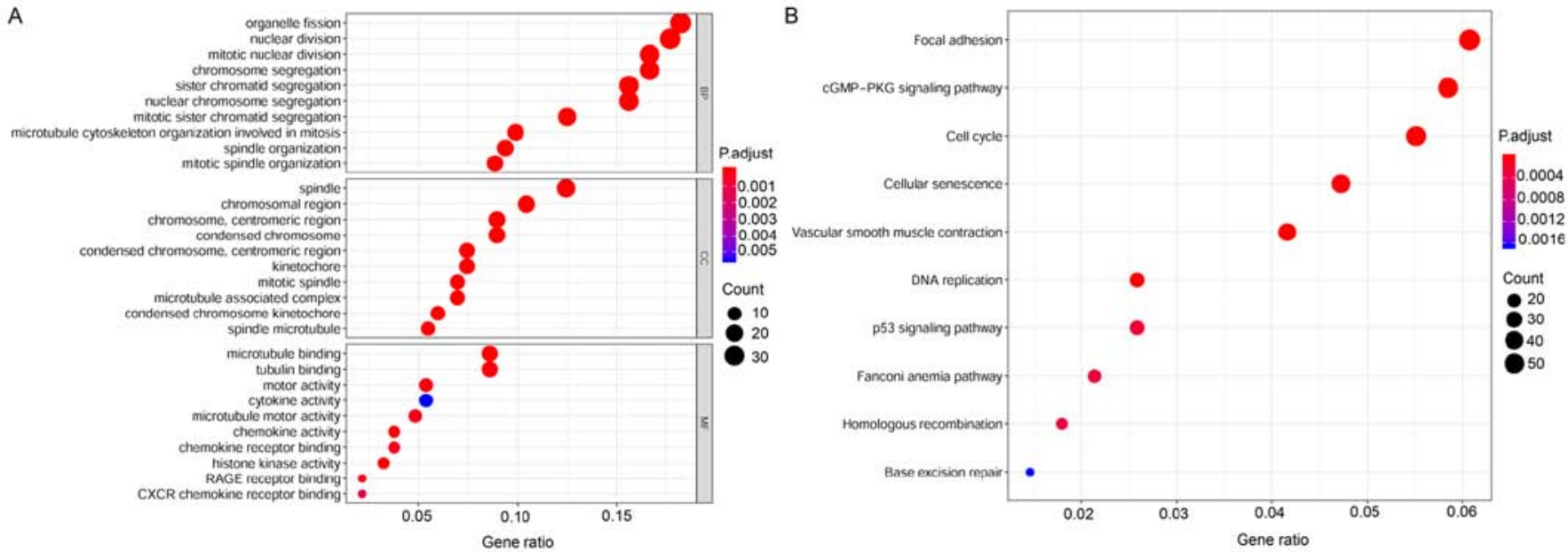

Figure 4. GO and KEGG pathway analyses. (A) The top 30 significantly enriched GO terms of DEmRNAs. (B) The top 10 enriched KEGG pathways of DEmRNAs. DE, differentially expressed. GO, gene ontology; KEGG, Kyoto Encyclopedia of Genes and Genomes.

were identified to be significantly associated with overall survival and 15 DEmiRNAs were closely linked to prognosis in CESC. Among the top 9 mRNAs that affected overall survival in CESC, C9orf84, CBX7, CCDC171, CSK, HELLS, and RIBC2 were positively associated with overall survival, whereas FASN, LATS2, and SMYD2 were negatively associated with survival (Fig. 6). Overexpressed DEmiRNAs, including miR-99a-5p, miR-20b-3p, miR-101-3p, miR-145-5p, miR-142-3p, miR-218-5p, miR-362-5p, miR-185-3p, miR-505-5p, miR-642a-5p, and miR-29b-2-5p, were revealed to be strongly associated with prolonged patient survival time, and upregulated DEmiRNAs, including miR-210-5p, miR-425-5p, miR-3613-5p, and miR-6892-5p, were associated with poor patient prognosis. The top 9 DEmiRNAs associated with overall survival are shown in Fig. 7.

Construction of ceRNA network in CESC. Competing endogenous RNA network analysis was based on three criteria (see the Materials and methods section) to determine the competing endogenous interactions among mRNAs and lncRNAs. A 


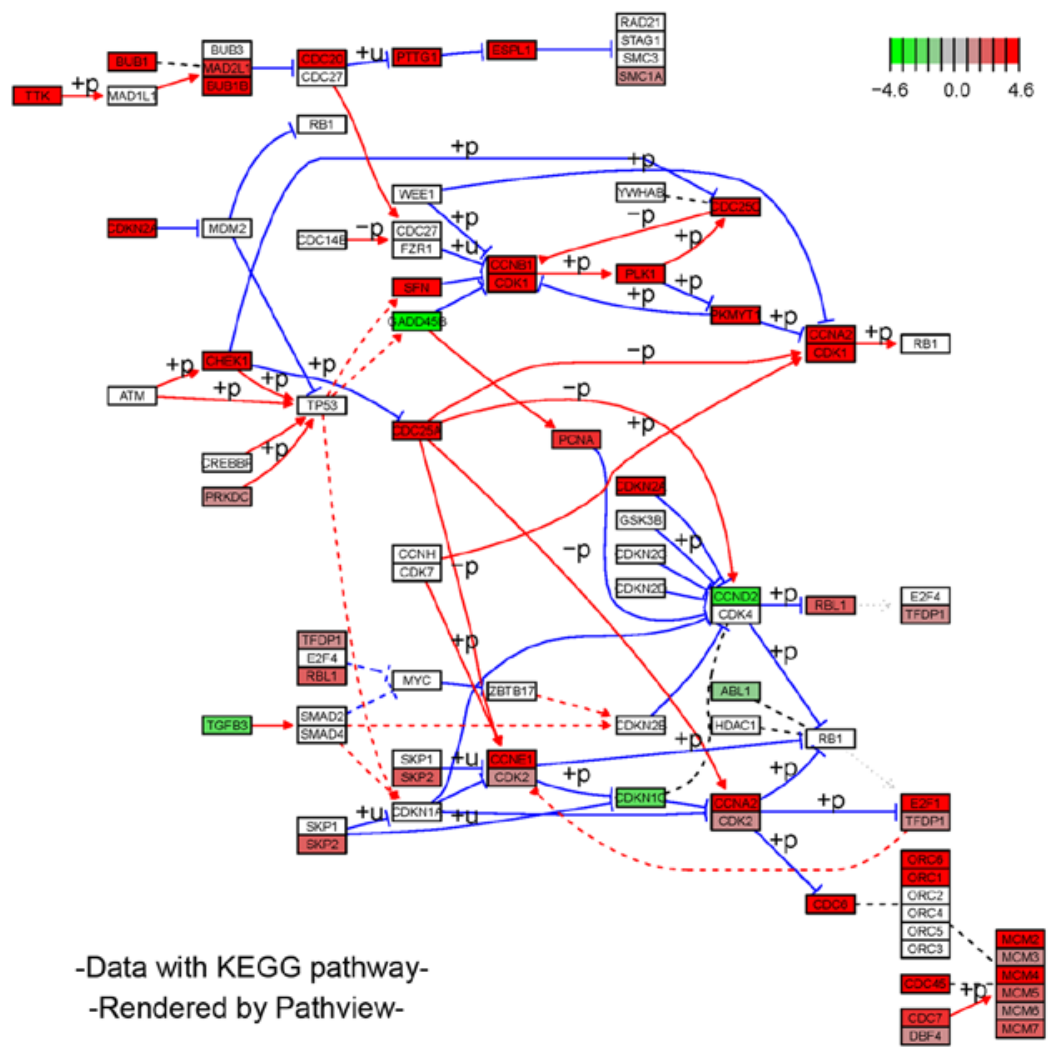

Edge types

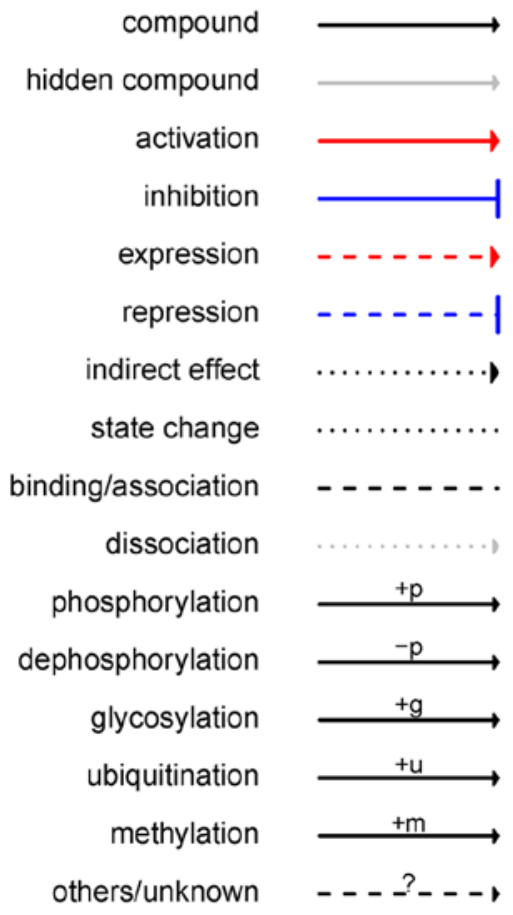

Figure 5. A plot of the cell cycle involving DEmRNAs in CESC. mRNAs with high and low expression are represented by red and green, respectively. The intensity of gradient color indicates expression level of the dysfunctional gene. CESC, cervical squamous cell carcinoma and endocervical adenocarcinoma; DE, differentially expressed.
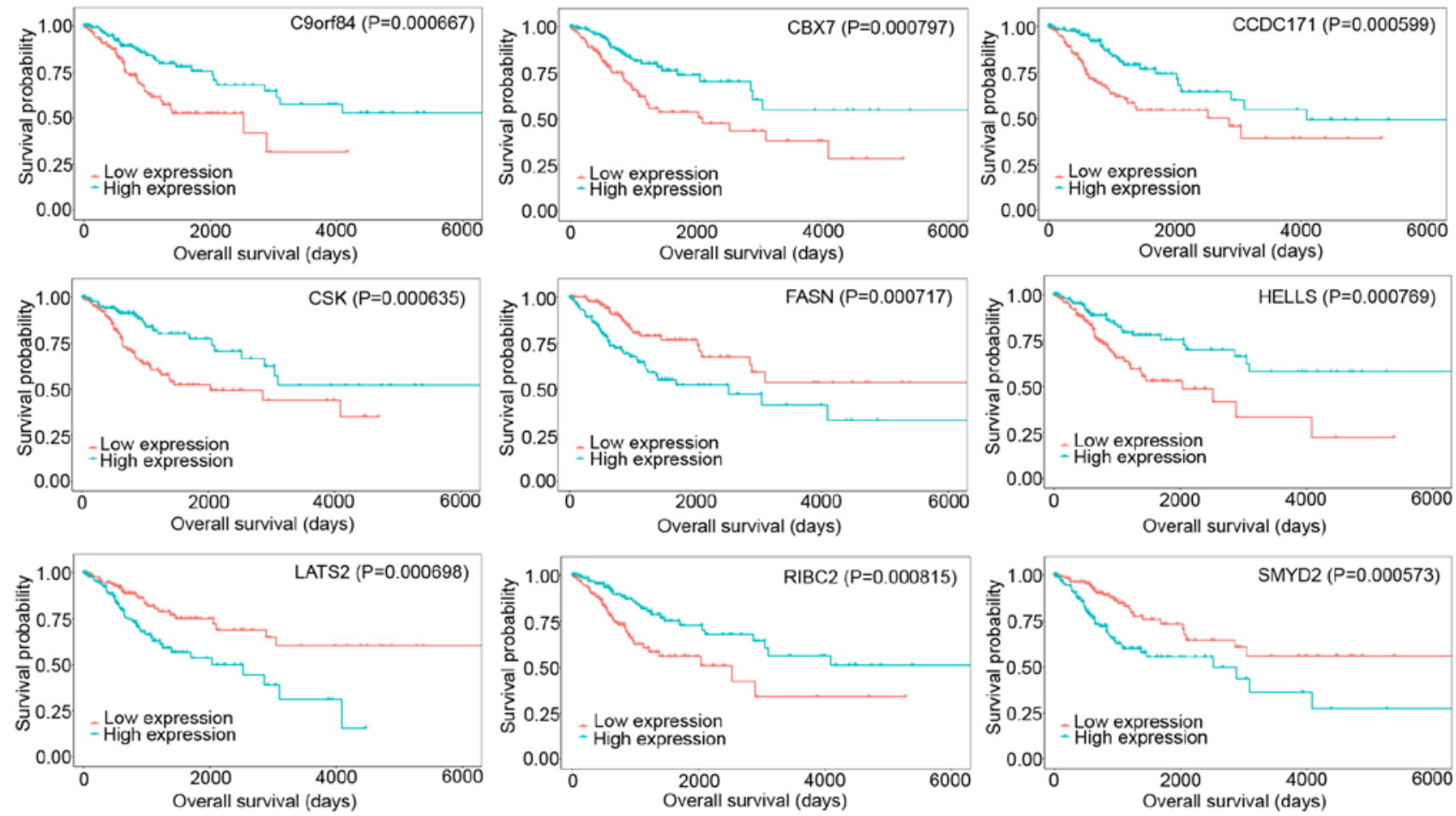

Figure 6. Kaplan-Meier survival curves of the top 9 DEmRNAs associated with overall survival. DE, differentially expressed.

hypergeometric test of shared miRNAs, expression correlation analysis of IncRNA-mRNA pairs, and regulation pattern analysis of shared miRNAs were all implemented to build a
ceRNA regulatory network of lncRNAs, miRNAs, and mRNAs in CESC. StarBase v2.0 was used to collect miRNA-lncRNA and/or miRNA-mRNA pairs for prediction and experimental 

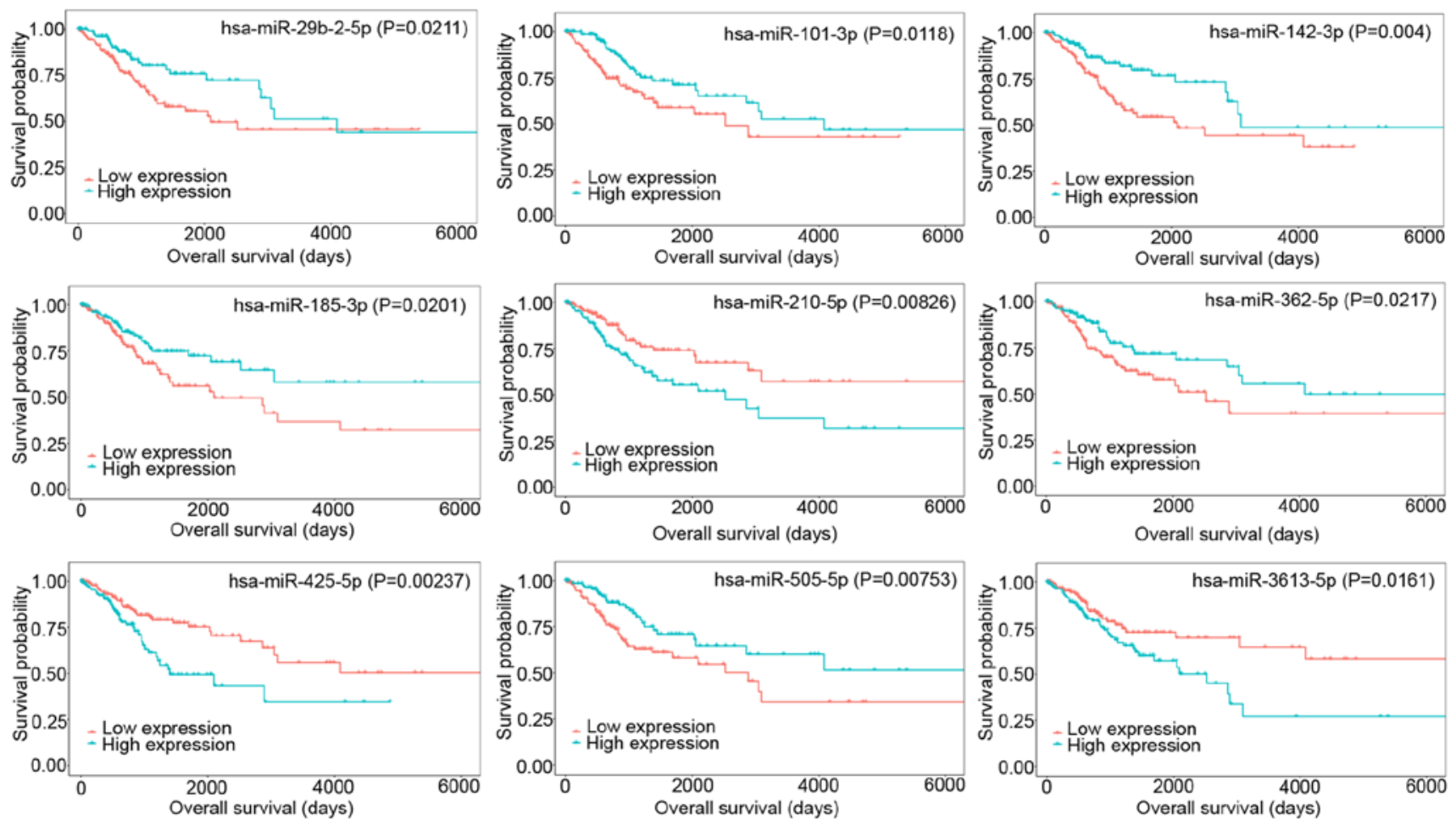

Figure 7. Kaplan-Meier analysis of the top 9 DEmiRNAs associated with overall survival. DE, differentially expressed.

A

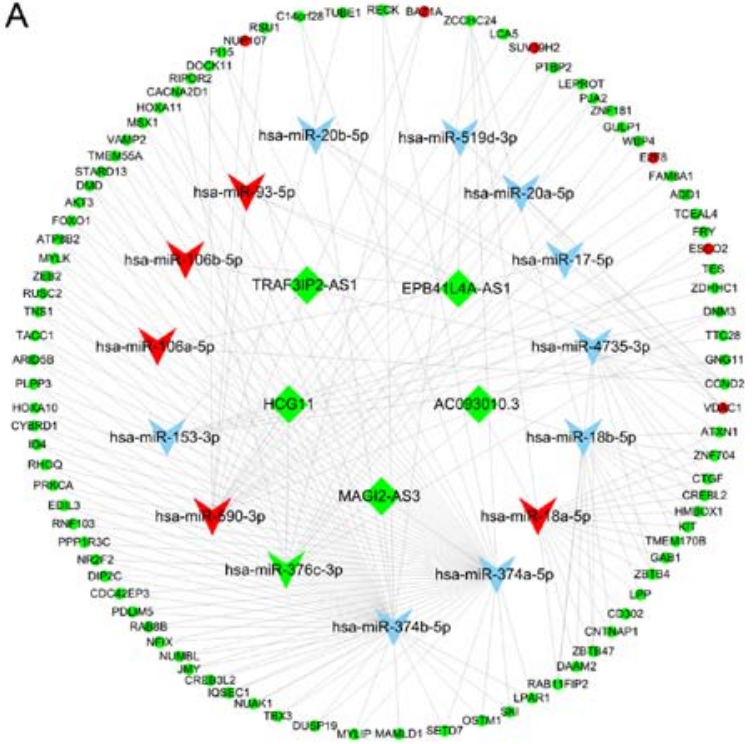

B

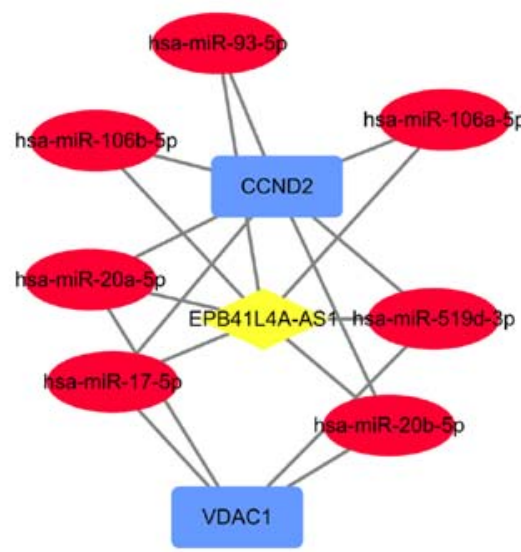

Figure 8. ceRNA networks in CESC. (A) The ceRNA network between DElncRNAs and DEmRNAs mediated by miRNAs. The round, rhombus, and triangle nodes represent mRNAs, IncRNAs, and miRNAs, respectively. Red indicates high expression, whereas green represents low expression. Blue triangle nodes represent miRNAs that do not belong to DEmiRNAs. (B) The subnetwork of lncRNA-miRNA-mRNA. Red ellipse, blue rectangles, and yellow diamonds represent miRNA, mRNA, and lncRNA, respectively. ceRNA, competitive endogenous RNA; CESC, cervical squamous cell carcinoma and endocervical adenocarcinoma; DE, differentially expressed.

verification. A co-expression network was constructed between the DElncRNAs and DEmRNAs and the network was further filtered using hyper P-value $<0.05$ and cor P-value $<0.05$. Cytoscape was used to visualize the ceRNA network. Ultimately, 110 nodes and 190 edges were identified, including 5 lncRNAs, 15 miRNAs, and 90 mRNAs, for the ceRNA network. The Cytoscape plug-in called 'cytoHubba' was used to detect hub nodes, and the MCC algorithm of cytoHubba was used to identify hub nodes. The top 10 genes were miR-374b-5p, miR-374a-5p, miR-590-3p, miR-18a-5p, miR-18b-5p, miR-4735-3p, miR-376c-3p, EPB41L4A-AS1, CCND2, and miR-153-3p, and their scores were 49, 48, 18, 17, 15, 10, 9, 7, 7, and 6, respectively. Among them, miR-18a-5p, miR-376c-3p, and miR-590-3p were included with the DEmiRNAs, whereas EPB41L4A-AS1 and CCND2 belonged to the DElncRNA and DEmRNA groups, respectively. Based on the MCC scores, a 
sub-network was established that centered around the IncRNA EPB41L4A-AS1, one of the top 10 most highly expressed genes (Fig. 8).

\section{Discussion}

LncRNAs are transcriptional products that are more than 200 nucleotides in length and do not appear to possess any significant coding protein function. LncRNAs are composed of various heterogeneous non-coding RNAs (15). Although lncRNAs are abundant among non-coding RNAs, their function has not been fully elucidated. Notably, there has been increasing evidence that IncRNAs significantly contribute to gene expression and regulation. In recent years, multiple studies have revealed that $\operatorname{lncRNAs}$ participate in various processes, including cell growth, cellular death, and epigenetic regulation (16). Yang et al (17), confirmed Gm16551 as an lncRNA regulator that suppresses lipogenesis and sterol regulatory element-binding protein 1c activity in the mouse liver. Moreover, the aberrant expression of lncRNAs is associated with pathogenesis and development of multiple carcinomas via activation of carcinogenic pathways and interaction with other RNAs (18).

The present study reported 60 upregulated and 90 downregulated lncRNAs among the IncRNAs that were differentially expressed between tumor tissues and normal samples. Notably, lncRNA MEG3 was found to be downregulated in our differential expression profile. It was previously identified using experimental techniques that the upregulation of MEG3 may inhibit cell growth through reducing the expression of miR-21-5p in carcinoma of the uterine cervix (19). The IncRNA C5orf66-AS1 was overexpressed among the DE RNAs. Rui et al (20) suggested that C5orf66-AS1 was markedly upregulated in uterine cervical neoplasms compared with its expression in paracervical tissues. LncRNA C5orf66-AS1 may regulate cancer proliferation and cell death in cervical cancer via sponging miR-637 as a ceRNA. In addition, IncRNA LINC00511 was also upregulated among aberrantly expressed lncRNAs. Lu et al (21), indicated that the high expression of LINC00511 was associated with poor disease outcome in breast cancer. In addition, the study demonstrated that LINC00511 interacted with miR-185-3p as a competing ceRNA to facilitate breast cancer tumorigenesis.

To explore the significance of DElncRNAs in expression profiles further, KM analysis was performed to detect the association between DElncRNAs and overall survival in patients with uterine cervix carcinoma. It was noted that 12 DElncRNAs were significantly associated with carcinoma outcome. The results of the study by He et al (22), were consistent with our findings, as the present study reported that high expression of IncRNA CASC15 was associated with poor disease outcome in hepatocellular carcinoma. Yin et al (23), demonstrated that AC016773.1 is an independent factor influencing survival in clear cell renal carcinoma via multivariate Cox regression analysis. The present study noted that some lncRNAs (AC008771.1, AC015871.3, AC019069.1, AC093278.2, and AL662844.4) that have been studied poorly to date are significantly associated with survival time, although further experimental verification is required for validation.

MiRNAs form one group of small non-coding RNA molecules that exist in animals, plants, and some viruses (24), and function in RNA silencing and post-transcription to regulate gene expression. Guay et al (25), suggested that transfer of miRNAs to rodent and human pancreatic $\beta$-cells results in chemokine expression and apoptosis of $\beta$-cells. Previous studies have demonstrated multiple roles for miRNAs in many biological processes. For example, Wüst et al (26), verified that loss of miR-1/133a hinders mitochondrial activity in skeletal muscle. Lee et al (27), demonstrated that epithelial microvesicle-containing cav-1/hnRNPA2B1 complex bound to miR-17/93-activated tissue macrophages and initiated an immune response. Furthermore, aberrant miRNA expression is implicated in disease states. Mehta et al (28), suggested that miR-132 may modulate B lymphopoiesis via Sox4 and inhibit development of cancerous B-cells. Singh et al (29), showed that miR-17 92-deficient type 2 innate lymphoid cells relieved lung inflammation after exposure to allergens in mice. MiRNAs may be upregulated or downregulated, and aberrantly expressed miRNAs are involved in tumorigenesis and the development of multiple types of cancer, including cervical cancer $(30,31)$.

In the present study, 133 miRNAs were differentially expressed between cervical tumors and normal solid samples. Among the DEmiRNAs, 80 miRNAs showed increased expression, and 53 miRNAs showed decreased expression, respectively. The data revealed that miRNA-205-5p, miRNA-210-5p, miRNA-425-5p, miRNA-21-5p, and miRNA-141-3p were highly expressed. Several studies have detected the relationship between these miRNAs and various types of cancer. Chen et al (32), showed that miRNA-205-5p may restrict the reproductive and invasive capabilities of colorectal carcinoma by inhibiting PTK7. Interestingly, miRNA-205-5p was the most highly expressed miRNA in our expression profiles, a finding which is consistent with those of Vilming Elgaaen et al (33), who reported that miRNA-205-5p was the most highly expressed $(\mathrm{FC}=74)$ miRNA in high-grade ovarian carcinoma. Qu et al (34), reported that miRNA-21-5p was highly expressed in pancreatic cancer, and further suggested a favorable sensitivity and specificity (0.77 and 0.80 , respectively) for the diagnostic value of miRNA-21-5p. Their study highlighted that miRNA-21-5p may be a prospective indicator in pancreatic cancer. It has also been observed that the expression of miR-141-3p is inversely associated with p53 expression in glioblastoma and normal brain tissues. MiR-141-3p facilitated glioma progression and temozolomide resistance by regulating p53 expression, and therefore may act as a new diagnostic and therapeutic indicator in glioblastoma (35). Hang et al (36), confirmed that miR-145-5p is strongly and negatively associated with connective tissue growth factor (CTGF) $(\mathrm{r}=0.1126, \mathrm{P}=0.02188)$, and downregulation of miR-145-5p was found to be associated with poor disease outcome and higher stages in ovarian cancer. The present study also found that miR-145-5p was one of the downregulated miRNAs. By comprehensively analyzing the relationship between DEmiRNAs and overall survival of patients with cervical cancer, miR-210-5p and miR-425-5p were found to be upregulated and had a significant negative association with overall survival. Ying et al (37), suggested that upregulation of miR-210-5p exerted an impact on carcinogenic signaling pathways, and may participate in the progression of kidney carcinoma. Low expression of miR-425-5p was 
indicated to inhibit cell growth, infiltration, and migration in gastric carcinoma. Moreover, downregulated miR-425-5p may suppress tumor metastasis to lung tissues in nude mice (38).

The importance of mRNAs in malignant neoplasms has been well studied. Therefore, the differences in mRNA expression between cervical cancer samples and normal tissues were analyzed. A total of 2,255 DEmRNAs were confirmed in our profile. KM analysis was simultaneously performed to explore the relationship between DEmRNAs and patient outcome. Fatty acid synthase gene (FASN) was determined to be highly expressed in cervical tumor samples, and an inverse association between FASN and overall survival was detected in patients with cervical cancer. Xia et al (39) demonstrated that FASN may serve as a potential prognostic biomarker in patients with cervical cancer through Cox regression analysis. Notably, the mRNAs CXCL12 and CCL21 were both downregulated in our data, and are known to be involved in invasion, migration, and progression of cervical cancer $(40,41)$. Moreover, a low expression of mRNA FOXO1 was observed, and it has been previously reported that downregulation of FOXO1 may induce cellular proliferation and activate tumor cell viability in carcinoma of the uterine cervix (42). Zhao et al (43) indicated that dysfunction of the mRNAs CHEK1 and CDKN2A, which were upregulated within our group of DEmRNAs, could facilitate cellular proliferation in cervical cancer.

The ceRNA network was originally proposed by Salmena et al (44) to highlight the notion that mRNAs, lncRNAs, and other RNAs may interact with miRNAs by sponging the miRNAs through MREs. Several studies have revealed that 1 cRNAs are able to indirectly regulate mRNAs through miRNAs $(45,46)$. Therefore, GO and KEGG analyses of DEmRNAs were conducted to provide insights into the impact of dysregulated lncRNAs. The enriched GO terms were mainly involved in cell division, cellular proliferation, cellular protein metabolic processes, and cell differentiation. The KEGG pathways of DEmRNAs were markedly enriched in tumor-associated pathways, including focal adhesion, cell cycle, cellular senescence, DNA replication, p53 signaling, Fanconi anemia, homologous recombination, and base excision repair. Moreover, the top $10 \mathrm{GO}$ terms enriched in DEmRNAs were primarily associated with the cell cycle in KEGG pathways.

Subsequently, the effects of DEmRNAs on overall survival were assessed, and 255 DEmRNAs with prognostic value in cervical cancer were identified. The DEmRNAs CBX7, SMYD2, and LATS2 were strongly associated with the clinical outcome of cervical cancer patients by KM analysis. Previous studies have shown that $\mathrm{CBX} 7$ is a vital tumor suppressor, and the absence of $\mathrm{CBX} 7$ contributes to tumorigenesis, whereas high expression of $\mathrm{CBX} 7$ is correlated with longer survival time $(47,48)$. High expression of SMYD2 was found in papillary thyroid carcinoma and hepatocellular carcinoma tissues, and patients with papillary thyroid cancer and hepatocellular cancer with upregulated SMYD2 were observed to have poor disease outcomes (49,50). Consistent with our findings, Luo et al (51) suggested that the expression of the mRNA LATS2 was a significant predictive factor of overall survival in lung adenocarcinoma.

A hypothesis of ceRNA network provided a new avenue for studies that could improve our understanding of the interac- tion between IncRNAs and mRNAs mediated by miRNAs. The construction of a IncRNA-miRNA-mRNA network offered new clues for revealing the key RNAs involved in tumorigenesis and progression of cervical cancer. Here, a miRNA-mediated IncRNA-miRNA-mRNA cross-talk network from TCGA database was generated. In the ceRNA network, there were 110 nodes and 190 edges, including 5 lncRNAs, 15 miRNAs, and 90 mRNAs. The scores of RNAs that were identified in the network were calculated, and a subnetwork was constructed that centered on the lncRNA EPB41L4A-AS1 in the top 10 genes based on MCC scores. The figure created for the ceRNA sub-network indicated that the IncRNA EPB41L4A-AS1 could indirectly interact with the mRNAs CCND2 and VDAC1 via miR-106a-5p, miR-93-5p, miR-106b-5p, miR-519d-3p, miR-17-5p, miR-20a-5p, and miR-20b-5p. This suggested that the lncRNA EPB41L4A-AS1 may function as a vital regulator in carcinoma of the uterine cervix. In line with this, Zhan et al (52) also provided evidence in support of EPB41L4A-AS1 acting as a potential candidate for stage progression and poor prognosis in ovarian cancer. It was shown in the present study how lncRNAs affect the expression of coding genes mediated by miRNAs, highlighting the importance of a lncRNA-miRNA-mRNA network in cervical cancer.

It is important to verify the function of the RNAs implicated in cervical cancer as well as the interaction among mRNAs, miRNAs, and lncRNAs, both in vitro and in vivo, using mice, for example, as an animal model. During in vivo research, the homology of mRNA, miRNA, and IncRNA must be considered. To answer this, it is necessary to obtain the mouse (or others) RNA-seq data and human RNA-seq data to check the homology via tools such as BLAST (Basic Local Alignment Search Tool). In the present study, RNA-seq profiles from mice or other animal models were not used, and would be useful to analyze in future studies.

In the literature, some mRNAs and miRNAs in mouse have functions that are similar to those of humans. For example, Qi et al (53) showed mRNA paired like homeodomain 1 (PITX1), which was overexpressed in the present study, can suppress both human telomerase reverse transcriptase (hTERT) and mouse TERT (mtert) promoter activity. Sastre-Perona et al (54) revealed that PITX1 is specifically expressed in basal tumor propagating cells, where it co-localizes with SOX2 and TRP63 and determines cell fate in mouse and human squamous cell carcinoma. Osei-Amponsa et al (55) confirmed a critical role for hypermethylation of FOXA1 in heterogeneity of bladder cancer, and epigenetic silencing of FOXA1 drives squamous differentiation in mice. In the present study, miRNA-205 was upregulated; Son et al (56) suggested that miRNA-205 of humans shares similar sequences to that of miRNA-712 in mice, and is highly conserved in most vertebrates. miRNA-205 and miRNA-712 also share $>50 \%$ of the cell signaling targets, including TIMP3. For the lncRNAs, this appears not to be the case. Further investigations into vertebrate lncRNAs revealed that, although lncRNAs are conserved in sequence, they are not conserved in terms of their transcription $(57,58)$. This means that, even when the sequence of a human IncRNA is conserved in another vertebrate species, there is often no transcription of a lncRNA in the orthologous genomic region. 
Our results have uncovered the potential mechanisms of tumorigenesis and prognosis in cervical cancer, and suggest that the IncRNA EPB41L4A-AS1 is both a novel and vital biomarker for carcinogenesis and progression, and also a candidate therapeutic target in cervical cancer that needs to be studied further. Drugs targeting lncRNA EPB41L4A-AS1, which is implicated in the overexpression of miR-106a/106b-5p, will have usefulness in the treatment of cervical cancer. However, to achieve this, further in vitro and in vivo experiments are needed to verify the mechanisms of mutual interaction between the IncRNA EPB41L4A-AS1 and related target genes in the ceRNA sub-network mentioned above.

In conclusion, in the present study, the expression profiles of IncRNA, miRNA, and mRNA in cervical cancer were analyzed from TCGA database. Cancer-specific RNAs that may be relevant for tumorigenesis, development, and prognosis were screened via differential gene expression analysis, KM survival analysis, and a comprehensive ceRNA network. Our study has significantly contributed to the current understand-ing of cervical cancer pathogenesis, and has identified novel RNAs as potential markers for diagnosis, prognosis, and therapy. Due to the limitations of bioinformatics analysis, however, large-scale prospective research will be necessary for further validation.

\section{Acknowledgements}

We are grateful to Mr Jiajia Xu and Ms Wenjing Xu (Shanghai Jieyi Biotechnology Co., Ltd, China) for their technical computer support.

\section{Funding}

No funding was received.

\section{Availability of data and materials}

The results in the present study are based upon data generated by the TCGA Research Network: https://www.cancer.gov/tcga. The datasets used and/or analyzed during the present study are available from the corresponding author on reasonable request.

\section{Authors' contributions}

PC and WZ designed the study and drafted the manuscript. YC, $\mathrm{XZ}$, and DY performed the data analysis. All authors read and approved the final manuscript.

\section{Ethics approval and consent to participate}

Not applicable.

\section{Patient consent for publication}

Not applicable.

\section{Competing interests}

The authors declare that they have no competing interests.

\section{References}

1. Waggoner SE: Cervical cancer. Lancet 361: 2217-2225, 2003.

2. Liu S, Zhang P, Chen Z, Liu M, Li X and Tang H: MicroRNA-7 downregulates XIAP expression to suppress cell growth and promote apoptosis in cervical cancer cells. FEBS Lett 587: 2247-2253, 2013

3. Wang H, Zhao Y, Chen M and Cui J: Identification of novel long non-coding and circular RNAs in human papillomavirus-mediated cervical cancer. Front Microbiol 8: 1720, 2017.

4. Li LJ, Zhao W, Tao SS, Leng RX, Fan YG, Pan HF and Ye DQ: Competitive endogenous RNA network: Potential implication for systemic lupus erythematosus. Expert Opin Ther Targets 21: 639-648, 2017.

5. Huarte M: The emerging role of lncRNAs in cancer. Nat Med 21: 1253-1261, 2015.

6. Yang R, Xing L, Wang M, Chi H, Zhang L and Chen J: Comprehensive analysis of differentially expressed profiles of lncRNAs/mRNAs and miRNAs with associated ceRNA networks in triple-negative breast cancer. Cell Physiol Biochem 50: 473-488, 2018.

7. Tomczak K, Czerwińska P and Wiznerowicz M: The Cancer Genome Atlas (TCGA): An immeasurable source of knowledge. Contemp Oncol (Pozn) 19 (1A): A68-A77, 2015.

8. Ritchie ME, Phipson B, Wu D, Hu Y, Law CW, Shi W and Smyth GK: limma powers differential expression analyses for RNA-sequencing and microarray studies. Nucleic Acids Res 43: e47, 2015.

9. Yu G, Wang LG, Han Y and He QY: clusterProfiler: An R package for comparing biological themes among gene clusters. OMICS 16: 284-287, 2012.

10. Furió-Tarí P, Tarazona S, Gabaldón T, Enright AJ and Conesa A: spongeScan: A web for detecting microRNA binding elements in lncRNA sequences. Nucleic Acids Res 44 (W1): W176-80, 2016.

11. Li JH, Liu S, Zhou H, Qu LH and Yang JH: starBase v2.0: Decoding miRNA-ceRNA, miRNA-ncRNA and protein-RNA interaction networks from large-scale CLIP-Seq data. Nucleic Acids Res 42 (D1): D92-D97, 2014.

12. Paci P, Colombo T and Farina L: Computational analysis identifies a sponge interaction network between long non-coding RNAs and messenger RNAs in human breast cancer. BMC Syst Biol 8: 83, 2014.

13. Shannon P, Markiel A, Ozier O, Baliga NS, Wang JT, Ramage D, Amin N, Schwikowski B and Ideker T: Cytoscape: A software environment for integrated models of biomolecular interaction networks. Genome Res 13: 2498-2504, 2003.

14. Chin $\mathrm{CH}$, Chen $\mathrm{SH}, \mathrm{Wu} \mathrm{HH}$, Ho CW, Ko MT and Lin CY: cytoHubba: Identifying hub objects and sub-networks from complex interactome. BMC Syst Biol 8 (Suppl 4): S11, 2014.

15. Zhang H, Chen Z, Wang X, Huang Z, He Z and Chen Y: Long non-coding RNA: A new player in cancer. J Hematol Oncol 6: 37, 2013.

16. $\mathrm{Hu} \mathrm{W}$, Alvarez-Dominguez JR and Lodish HF: Regulation of mammalian cell differentiation by long non-coding RNAs. EMBO Rep 13: 971-983, 2012.

17. Yang L, Li P, Yang W, Ruan X, Kiesewetter K, Zhu J and Cao H: Integrative transcriptome analyses of metabolic responses in mice define pivotal LncRNA metabolic regulators. Cell Metab 24: 627-639, 2016.

18. Bolha L, Ravnik-Glavač M and Glavač D: Long noncoding RNAs as biomarkers in cancer. Dis Markers 2017: 7243968, 2017.

19. Zhang J, Yao T, Wang Y, Yu J, Liu Y and Lin Z: Long noncoding RNA MEG3 is downregulated in cervical cancer and affects cell proliferation and apoptosis by regulating miR-21. Cancer Biol Ther 17: 104-113, 2016.

20. Rui X, Xu Y, Jiang X, Ye W, Huang Y and Jiang J: Long non-coding RNA C5orf66-AS1 promotes cell proliferation in cervical cancer by targeting miR-637/RING1 axis. Cell Death Dis 9: 1175, 2018.

21. Lu G, Li Y, Ma Y, Lu J, Chen Y, Jiang Q, Qin Q, Zhao L, Huang Q, Luo Z, et al: Long noncoding RNA LINC00511 contributes to breast cancer tumourigenesis and stemness by inducing the miR-185-3p/E2F1/Nanog axis. J Exp Clin Cancer Res 37: 289, 2018.

22. He T, Zhang L, Kong Y, Huang Y, Zhang Y, Zhou D, Zhou X, Yan Y, Zhang L, Lu S, et al: Long non-coding RNA CASC15 is upregulated in hepatocellular carcinoma and facilitates hepatocarcinogenesis. Int J Oncol 51: 1722-1730, 2017.

23. Yin $\mathrm{H}$, Wang $\mathrm{X}$, Zhang X, Wang Y, Zeng Y, Xiong Y, Li T, Lin R, Zhou Q, Ling H, et al: Integrated analysis of long noncoding RNA associated-competing endogenous RNA as prognostic biomarkers in clear cell renal carcinoma. Cancer Sci 109: 3336-3349, 2018. 
24. Ambros V: The functions of animal microRNAs. Nature 431: 350-355, 2004.

25. Guay C, Kruit JK, Rome S, Menoud V, Mulder NL, Jurdzinski A, Mancarella F, Sebastiani G, Donda A, Gonzalez BJ, et al: Lymphocyte-derived exosomal microRNAs promote pancreatic $\beta$ cell death and may contribute to type 1 diabetes development. Cell Metab 29: 348-361.e6, 2019.

26. Wüst S, Dröse S, Heidler J, Wittig I, Klockner I, Franko A Bonke E, Günther S, Gärtner U, Boettger T, et al: Metabolic maturation during muscle stem cell differentiation is achieved by miR-1/133a-mediated inhibition of the Dlk1-Dio3 mega gene cluster. Cell Metab 27: 1026-1039.e6, 2018.

27. LeeH,LiC,Zhang Y,Zhang D, Otterbein LE and Jin Y: Caveolin-1 selectively regulates microRNA sorting into microvesicles after noxious stimuli. J Exp Med 216: 2202-2220, 2019.

28. Mehta A, Mann M, Zhao JL, Marinov GK, Majumdar D, Garcia-Flores Y, Du X, Erikci E, Chowdhury K and Baltimore D: The microRNA-212/132 cluster regulates B cell development by targeting Sox4. J Exp Med 212: 1679-1692, 2015.

29. Singh PB, Pua HH, Happ HC, Schneider C, von Moltke J, Locksley RM, Baumjohann D and Ansel KM: MicroRNA regulation of type 2 innate lymphoid cell homeostasis and function in allergic inflammation. J Exp Med 214: 3627-3643, 2017.

30. Giza DE, Vasilescu C and Calin GA: MicroRNAs and ceRNAs: Therapeutic implications of RNA networks. Expert Opin Biol Ther 14: 1285-1293, 2014.

31. Pardini B, De Maria D, Francavilla A, Di Gaetano C, Ronco G and Naccarati A: MicroRNAs as markers of progression in cervical cancer: A systematic review. BMC Cancer 18: 696, 2018

32. Chen S, Wang Y, Su Y, Zhang L, Zhang M, Li X, Wang J and Zhang X: miR-205-5p/PTK7 axis is involved in the proliferation migration and invasion of colorectal cancer cells. Mol Med Rep 17: 6253-6260, 2018.

33. Vilming Elgaaen B, Olstad OK, Haug KB, Brusletto B, Sandvik L, Staff AC, Gautvik KM and Davidson B: Global miRNA expression analysis of serous and clear cell ovarian carcinomas identifies differentially expressed miRNAs including miR-200c-3p as a prognostic marker. BMC Cancer 14: 80, 2014.

34. Qu K, Zhang X, Lin T, Liu T, Wang Z, Liu S, Zhou L, Wei J, Chang H, Li K, et al: Circulating miRNA-21-5p as a diagnostic biomarker for pancreatic cancer: Evidence from comprehensive miRNA expression profiling analysis and clinical validation. Sci Rep 7: 1692, 2017.

35. Zhou X, Wu W, Zeng A, Nie E, Jin X, Yu T, Zhi T, Jiang K, Wang Y, Zhang J, et al: MicroRNA-141-3p promotes glioma cell growth and temozolomide resistance by directly targeting p53. Oncotarget 8: 71080-71094, 2017.

36. Hang W, Feng Y, Sang Z, Yang Y, Zhu Y, Huang Q and Xi X: Downregulation of miR-145-5p in cancer cells and their derived exosomes may contribute to the development of ovarian cancer by targeting CT. Int J Mol Med 43: 256-266, 2019.

37. Ying $G$, Wu R, Xia M, Fei X, He QE, Zha C and Wu F. Identification of eight key miRNAs associated with renal cell carcinoma: A meta-analysis. Oncol Lett 16: 5847-5855, 2018.

38. Zhang Z, Li Y, Fan L, Zhao Q, Tan B, Li Z and Zang A: microRNA-425-5p is upregulated in human gastric cancer and contributes to invasion and metastasis in vitro and in vivo. Exp Ther Med 9: 1617-1622, 2015.

39. Xia L, Wang H, Cai S, Su X, Shen J, Meng Q, Chen Y, Li L, Yan J, Zhang C, et al: Integrated analysis of a competing endogenous RNA network revealing a prognostic signature for cervical cancer. Front Oncol 8: 368, 2018.

40. Teicher BA and Fricker SP: CXCL12 (SDF-1)/CXCR4 pathway in cancer. Clin Cancer Res 16: 2927-2931, 2010.

41. Xiong Y, Huang F, Li X, Chen Z, Feng D, Jiang H, Chen W and Zhang X: CCL21/CCR7 interaction promotes cellular migration and invasion via modulation of the MEK/ERK1/2 signaling pathway and correlates with lymphatic metastatic spread and poor prognosis in urinary bladder cancer. Int J Oncol 51: 75-90, 2017.

42. Prasad SB, Yadav SS, Das M, Govardhan HB, Pandey LK, Singh S, Pradhan S and Narayan G: Down regulation of FOXO1 promotes cell proliferation in cervical cancer. J Cancer 5 : $655-662,2014$
43. Zhao L, Zhang Z, Lou H, Liang J, Yan X, Li W, Xu Y and Ou R: Exploration of the molecular mechanisms of cervical cancer based on mRNA expression profiles and predicted microRNA interactions. Oncol Lett 15: 8965-8972, 2018

44. Salmena L, Poliseno L, Tay Y, Kats L and Pandolfi PP: A ceRNA hypothesis: The Rosetta Stone of a hidden RNA language? Cell 146: 353-358, 2011

45. Li F, Huang C, Li Q and Wu X: Construction and comprehensive analysis for dysregulated long non-coding RNA (lncRNA)-associated competing endogenous RNA (ceRNA) network in gastric cancer. Med Sci Monit 24: 37-49, 2018.

46. Song J, Ye A, Jiang E, Yin X, Chen Z, Bai G, Zhou Y and Liu J: Reconstruction and analysis of the aberrant lncRNA-miRNA-mRNA network based on competitive endogenous RNA in CESC. J Cell Biochem 119: 6665-6673, 2018.

47. Ni S, Wang H, Zhu X, Wan C, Xu J, Lu C, Xiao L, He J, Jiang C, Wang W, et al: CBX7 suppresses cell proliferation, migration, and invasion through the inhibition of PTEN/Akt signaling in pancreatic cancer. Oncotarget 8: 8010-8021, 2017.

48. Forzati F, Federico A, Pallante P, Abbate A, Esposito F, Malapelle U, Sepe R, Palma G, Troncone G, Scarfò M, et al: CBX7 is a tumor suppressor in mice and humans. J Clin Invest 122: 612-623, 2012.

49. Xu W, Chen F, Fei X, Yang X and Lu X: Overexpression of SET and MYND domain-containing protein 2 (SMYD2) is associated with tumor progression and poor prognosis in patients with papillary thyroid carcinoma. Med Sci Monit 24: 7357-7365, 2018

50. Zuo SR, Zuo XC, He Y, Fang WJ, Wang CJ, Zou H, Chen P, Huang LF, Huang LH, Xiang $\mathrm{H}$, et al: Positive expression of SMYD2 is associated with poor prognosis in patients with primary hepatocellular carcinoma. J Cancer 9: 321-330, 2018

51. Luo SY, Sit KY, Sihoe AD, Suen WS, Au WK, Tang X, Ma ES, Chan WK, Wistuba II, Minna JD, et al: Aberrant large tumor suppressor 2 (LATS2) gene expression correlates with EGFR mutation and survival in lung adenocarcinomas. Lung Cancer 85: 282-292, 2014.

52. Zhan L, Li J and Wei B: Long non-coding RNAs in ovarian cancer. J Exp Clin Cancer Res 37: 120, 2018.

53. Qi DL, Ohhira T, Fujisaki C, Inoue T, Ohta T, Osaki M, Ohshiro E, Seko T, Aoki S, Oshimura M, et al: Identification of PITX1 as a TERT suppressor gene located on human chromosome 5. Mol Cell Biol 31: 1624-1636, 2011.

54. Sastre-Perona A, Hoang-Phou S, Leitner MC, Okuniewska M, Meehan S and Schober M: De novo PITX1 expression controls bi-stable transcriptional circuits to govern self-renewal and differentiation in squamous cell carcinoma. Cell Stem Cell 24: 390-404.e8, 2019.

55. Osei-Amponsa V, Buckwalter JM, Shuman L, Zheng Z, Yamashita H, Walter V, Wildermuth T, Ellis-Mohl J, Liu C, Warrick JI, et al: Hypermethylation of FOXA1 and allelic loss of PTEN drive squamous differentiation and promote heterogeneity in bladder cancer. Oncogene 39: 1302-1317, 2020.

56. Son DJ, Kumar S, Takabe W, Kim CW, Ni CW, Alberts-Grill N, Jang IH, Kim S, Kim W, Won Kang S, et al: The atypical mechanosensitive microRNA-712 derived from pre-ribosomal RNA induces endothelial inflammation and atherosclerosis. Nat Commun 4: 3000, 2013

57. Washietl S, Kellis M and Garber M: Evolutionary dynamics and tissue specificity of human long noncoding RNAs in six mammals. Genome Res 24: 616-628, 2014.

58. Kutter C, Watt S, Stefflova K, Wilson MD, Goncalves A, Ponting CP, Odom DT and Marques AC: Rapid turnover of long noncoding RNAs and the evolution of gene expression. PLoS Genet 8: e1002841, 2012.

This work is licensed under a Creative Commons Attribution-NonCommercial-NoDerivatives 4.0 International (CC BY-NC-ND 4.0) License. 